\title{
Correction: Cytoplasmic incompatibility and host population
} \section{structure}

\section{J. Engelstädter $\cdot$ A. Telschow}

Published online: 24 November 2020

(c) The Genetics Society 2020

\section{Correction to: Heredity}

https://doi.org/10.1038/hdy.2009.53

$$
\hat{p}_{3}=\frac{1}{A}\left(1-f+l_{C I}+\sqrt{\left(1-f+l_{C I}\right)^{2}-2 A(1-f t}\right)
$$

In the original PDF version of this Article, in Box 1, Eq. (2), incorrect formulae were given for the equilibria $\hat{p}_{2}$ and $\hat{p}_{3}$. The correct formulae are:

$$
\begin{aligned}
& \hat{p}_{1}=0 \\
& \hat{p}_{2}=\frac{1}{A}\left(1-f+l_{C I}-\sqrt{\left(1-f+l_{C I}\right)^{2}-2 A(1-f t}\right),
\end{aligned}
$$

where $A=2 l_{C I}(1-f+f t)$. The error only concerns the formulae themselves; the figures in this box as well as the interpretations remain valid.

The authors would like to thank Dr Petteri Karisto for bringing this error to their attention and would like to apologise to all readers for any inconvenience caused. 